\title{
Finite-Time Stability Analysis of Discrete-Time Linear Singular Systems
}

\author{
Songlin Wo and Xiaoxin Han \\ School of Electrical and Information Engineering, Jiangsu University of Technology, Changzhou, Jiangsu 213001, China \\ Correspondence should be addressed to Songlin Wo; wosonglin2000@aliyun.com
}

Received 23 October 2013; Accepted 24 December 2013; Published 9 January 2014

Academic Editor: Zhengguang Wu

Copyright ( 2014 S. Wo and X. Han. This is an open access article distributed under the Creative Commons Attribution License, which permits unrestricted use, distribution, and reproduction in any medium, provided the original work is properly cited.

\begin{abstract}
The finite-time stability (FTS) problem of discrete-time linear singular systems (DTLSS) is considered in this paper. A necessary and sufficient condition for FTS is obtained, which can be expressed in terms of matrix inequalities. Then, another form of the necessary and sufficient condition for FTS is also given by using matrix-null space technology. In order to solve the stability problem expediently, a sufficient condition for FTS is given via linear matrix inequality (LMI) approach; this condition can be expressed in terms of LMIs. Finally, an illustrating example is also given to show the effectiveness of the proposed method.
\end{abstract}

\section{Introduction}

It is well known that singular systems (which are also known as descriptor systems and differential-algebraic systems) have started to play an important role in the fields of engineering such as power systems, economical systems, robotic systems, and chemical processes. One of the most important research areas of singular systems is Lyapunov stability and stabilization. In the last two decades, the problems of stability analysis and control of singular discrete-time systems have attracted a lot of attention. Various approaches have been made to deal with these issues and significant advances have been made on these topics in the literature (see, $[1-5]$ and references therein).

Lyapunov asymptotic stability (LAS) is enough for practical applications, but there are some cases where large values of the state are not acceptable, for instance in the presence of saturations. Recently, much attention has been focused on the problem of finite-time stability (FTS) (or short-time stability). A system is said to be finite-time stable if, once we fix a time-interval, its state does not exceed some bounds during this time-interval. In these cases, we need to check that these unacceptable values are not attained by the state; for these purposes, FTS could be used. Some early results of FTS for state-space system can be found in [6, 7]; more recently, the concept of FTS for state-space system has been revisited in the light of recent results coming from LMI theory, which has allowed finding less conservative conditions guaranteeing FTS, finite-time bounded (FTB), and finite-time stabilization of uncertain, linear continuous-time systems [8-10]. The discrete-time case is dealt with in the paper [11-15], where sufficient conditions for finite-time stability, expressed in terms of matrix inequalities or LMIs, are given. More recently, other contributions to FTS for discrete-time system have been given in [16-19]. So far, however, compared with numerous research results of FTS for state-space systems, few results of finite-time stability of singular system have been given in the literature.

In this paper, which is the extended version of [14] to singular systems, we consider the FTS problem of DTLSS. Our main analysis theorem guarantees FTS if and only if either an invertible symmetric matrix function solving a certain Lyapunov matrix inequality exists or a positive matrix function and a symmetric matrix solving a certain LMIs are satisfied. The condition solving a certain Lyapunov matrix inequality cannot be used as the starting point to solve the synthesis problem. Therefore, in view of the design problem, we focus on the condition involving LMI. However, this condition can become computationally hard to apply, since it requires studying the feasibility of $N$ difference inequalities, if $[1, N]$ is the time interval in which FTS is studied. For this reason a sufficient condition for FTS which requires checking the feasibility of only one inequality is given. 
The rest of the paper is organized as follows. The problem statement and definitions are introduced in Section 2. In Section 3, the main results of the conditions of finitetime stability of discrete-time singular systems are given. In Section 4, one simulation example is given to illustrate the theoretical result. Finally, conclusion is given in Section 5.

Notation. Throughout this paper, for real symmetric matrices $X$ and $Y$, the notation $X \geq Y$ (resp., $X>Y$ ) means that the matrix $X-Y$ is positive semi-definite (resp., positive definite). $I$ is the identity matrix with appropriate dimension. The superscript " $T$ " represents the transpose. Matrices, if not explicitly stated, are assumed to have compatible dimensions. $\operatorname{Rank} X$ represents the rank of matrix $X$.

\section{Problem Statement and Preliminaries}

In this paper, we consider the following discrete-time linear singular systems (DTLSS):

$$
E x(k+1)=A x(k),
$$

where $x(k) \in R^{n}$ is the state. The matrix $E \in R^{n \times n}$ has $\operatorname{rank} E=$ $r(r \leq n)$ and $A$ takes value in $R^{n \times n}$, respectively. The general idea of finite-time stability of singular system concerns the stability of the state of the system over a finite time interval for some given initial conditions; this concept can be formalized through the following definition.

Definition 1. The discrete-time linear singular system (1) is said to be regular if $\operatorname{det}(s E-A)$ is not identically zero.

Definition 2. The discrete-time linear singular system (1) is said to be causal if system (1) is regular and $\operatorname{deg}(\operatorname{det}(s E-A))=$ $\operatorname{rank} E$.

Definition 3 (finite-time stability, FTS). The discrete-time linear singular system (1) is said to be regular, causal, and finite-time stable with respect to $(\varepsilon, R, N)$, where $R$ is a positive definite matrix and $N \in N_{+}$if DTLSS (1) is regular, causal and

$$
\begin{array}{r}
x^{T}(0) E^{T} R E x(0) \leq 1 \Longrightarrow x^{T}(k) E^{T} R E x(k)<\varepsilon^{2} \\
\forall k \in\{1,2, \ldots, N\} .
\end{array}
$$

Remark 4. Lyapunov asymptotic stability (LAS) and FTS are independent concepts: a system in which FTS may not be LAS; conversely a LAS system could not be FTS if, during the transients, its state exceeds the prescribed bounds. used.

In the proof of the theorem, the following results will be

Lemma 5 (see [20]). The matrix measure $\mu(X)$ of the matrix $X$ has following properties:
(a) $-\|X\| \leq \operatorname{Re} \lambda(X) \leq \mu(X) \leq\|X\|$
(b) $\mu(X)=(1 / 2) \lambda_{\max }\left(X+X^{T}\right)$.

Lemma 6 (see [14]). The following statements are equivalent.

(i) System $x(k+1)=A x(k)$ is FTS with respect to $(\varepsilon, R, N)$.

(ii) For each $k \in\{1,2, \ldots, N\}$, there exists a symmetric matrix-valued function

$$
P_{k}(\cdot): h \in\{0,1,2, \ldots, k\} \longmapsto P_{k}(h) \in R^{n \times n},
$$

such that

$$
\begin{gathered}
A^{T} P_{k}(h+1) A-P_{k}(h)<0, \\
h \in\{0,1,2, \ldots, k-1\} ; \\
P_{k}(k) \geq R, \\
P_{k}(0) \leq \varepsilon^{2} R .
\end{gathered}
$$

The main goal of the paper is to find some conditions which guarantee that the system (1) is regular, causal, and FTS.

\section{Main Results}

Firstly, by using matrix inequalities approach, we give a necessary and sufficient condition under which DTLSS (1), without assumption of regularity, will be regular, causal, and finite-time stable with respect to $(\varepsilon, R, N)$.

Theorem 7. The following two statements are equivalent.

(i) The DTLSS (1) is regular, causal, and FTS with respect to $(\varepsilon, R, N)$.

(ii) For each $k \in\{1,2, \ldots, N\}$, there exists an invertible symmetric matrix-valued function

$$
P_{k}(\cdot): h \in\{0,1,2, \ldots, k\} \longmapsto P_{k}(h) \in R^{n \times n},
$$

such that

$$
\begin{gathered}
A^{T} P_{k}(h+1) A-E^{T} P_{k}(h) E<0, \\
h \in\{0,1,2, \ldots, k-1\} ; \\
E^{T} P_{k}(k) E \geq E^{T} R E, \\
E^{T} P_{k}(0) E \leq \varepsilon^{2} E^{T} R E .
\end{gathered}
$$

Proof. Firstly, we prove that (ii) implies (i). Let $k \in\{1,2, \ldots$, $N\}$, and assume there exists an invertible symmetric matrixvalued $P_{k}(\cdot)$ such that (6a), (6b), and (6c) hold. Let $\widetilde{M}, \widetilde{N} \in$ $R^{n \times n}$ be nonsingular matrices such that

$$
\widetilde{M} E \widetilde{N}=\left(\begin{array}{cc}
I_{r} & 0 \\
0 & 0
\end{array}\right),
$$

where $I_{r}$ is an identity matrix with $r \times r$ dimensions. 
New partitions $\widetilde{M}^{-T} P_{k}(h) \widetilde{M}^{-1}$ and $\widetilde{M} A \widetilde{N}$ conform to $\widetilde{M} E \widetilde{N}$; that is,

$$
\begin{aligned}
\widetilde{M}^{-T} P_{k}(h) \widetilde{M}^{-1} & =\left(\begin{array}{ll}
P_{1 k}(h) & P_{2 k}(h) \\
P_{2 k}^{T}(h) & P_{3 k}(h)
\end{array}\right), \\
\widetilde{M} A \widetilde{N} & =\left(\begin{array}{ll}
A_{1} & A_{2} \\
A_{3} & A_{4}
\end{array}\right) .
\end{aligned}
$$

From (6b), (7), and (8), it is easy to show that $P_{1 k}(k) \geq 0$. By using (6a) together with (7) and (8), we have

$$
\left(\begin{array}{cc}
* & * \\
* & A_{2}^{T} P_{1 k}(k) A_{2}+H+H^{T}
\end{array}\right)<0
$$

where $H=A_{2}^{T} P_{2 k}(k) A_{4}+(1 / 2) A_{4}^{T} P_{3 k}(k) A_{4}$. “*” represents a matrix not used in the following discussion.

Taking (9) into account and noting that $P_{1 k}(k) \geq 0$, we get $H+H^{T}<0$. By Lemma 5 ,

$$
\begin{aligned}
& \operatorname{Re}\left(\left(A_{2}^{T} P_{2 k}(k)+\frac{1}{2} A_{4}^{T} P_{3 k}(k)\right) A_{4}\right) \\
& \quad=\operatorname{Re}(H) \leq \mu(H)=\frac{1}{2} \lambda_{\max }\left(H+H^{T}\right)<0 .
\end{aligned}
$$

Then it can be easily shown that $\left(A_{2}^{T} P_{2 k}(k)+\right.$ $\left.(1 / 2) A_{4}^{T} P_{3 k}(k)\right) A_{4}$ is invertible, which implies that $A_{4}$ is invertible too. Hence, in the light of definition and the results in [21], we can conclude that the DTLSS (1) is regular and causal.

On the other hand, by (6a) we can draw

$$
\begin{gathered}
x^{T}(h+1) E^{T} P_{k}(h+1) E x(h+1)-x^{T}(h) E^{T} P_{k}(h) E x(h) \\
=x^{T}(h)\left(A^{T} P_{k}(h+1) A-E^{T} P_{k}(h) E\right) x(h)<0 .
\end{gathered}
$$

By summing (11) between 0 and $k-1$, we can draw a conclusion that

$$
x^{T}(k) E^{T} P_{k}(k) E x(k)-x^{T}(0) E^{T} P_{k}(0) E x(0)<0 .
$$

From the last inequality and by using (6b) and (6c), it follows that

$$
\begin{aligned}
x^{T}(k) E^{T} R E x(k) & \leq x^{T}(k) E^{T} P_{k}(k) E x(k) \\
& <x^{T}(0) E^{T} P_{k}(0) E x(0) \\
& \leq \varepsilon^{2} x^{T}(0) E^{T} R E x(0) .
\end{aligned}
$$

Therefore, if $x^{T}(0) E^{T} R E x(0) \leq 1$, we conclude that $x^{T}(k) E^{T} R E x(k)<\varepsilon^{2}$ for all $k \in\{1,2, \ldots, N\}$; that is, the DTLSS (1) is regular, causal, and FTS with respect to $(\varepsilon, R, N)$.

Secondly, we prove that (i) implies (ii). Now let us assume that the DTLSS (1) is regular, causal, and FTS with respect to $(\varepsilon, R, N)$. Then by continuity arguments, it follows that there exists a sufficiently small $\gamma>0$ and matrices $\widehat{M}, \widehat{N} \in R^{n \times n}$ are nonsingular matrices such that

$$
\widehat{M} E \widehat{N}=\left(\begin{array}{cc}
I_{r} & 0 \\
0 & 0
\end{array}\right), \quad \widehat{M} A \widehat{N}=\left(\begin{array}{cc}
A_{r} & 0 \\
0 & I_{n-r}
\end{array}\right),
$$

and letting $z(h)=\gamma \widehat{N}^{-1} x(h)$,

$$
x^{T}(0) E^{T} R E x(0) \leq 1 \Longrightarrow x^{T}(k) E^{T} R E x(k)+\|z\|_{2}^{2}<\varepsilon^{2},
$$

where $\|z\|_{2}^{2}=\sum_{h=0}^{k} z^{T}(h) z(h) . A_{r}$ is a matrix with $r \times r$ dimensions, and $I_{n-r}$ is an identity matrix with $(n-r) \times(n-r)$ dimensions.

New partitions $\widehat{M}^{-T} P_{k}(h) \widehat{M}^{-1}$ and $\widehat{M} R \widehat{N}$ conform to $\widehat{M} E \widehat{N}$; that is,

$$
\begin{aligned}
& \widehat{M}^{-T} P_{k}(h) \widehat{M}^{-1}=\left(\begin{array}{ll}
\widehat{P}_{1 k}(h) & \widehat{P}_{2 k}(h) \\
\widehat{P}_{2 k}^{T}(h) & \widehat{P}_{3 k}(h)
\end{array}\right), \\
& \widehat{M}^{-T} R \widehat{M}^{-1}=\left(\begin{array}{ll}
R_{1} & R_{2} \\
R_{3} & R_{4}
\end{array}\right) .
\end{aligned}
$$

Now let $P_{k}(\cdot): h \in\{0,1,2, \ldots, k\} \mapsto P_{k}(h) \in R^{n \times n}$ defined as follows:

$$
\begin{gathered}
A_{r}^{T} \widehat{P}_{1 k}(h+1) A_{r}=\widehat{P}_{1 k}(h)-\gamma^{2} I_{r}, \quad \widehat{P}_{1 k}(k)=R_{1}, \\
\widehat{P}_{2 k}(h)=0, \quad \widehat{P}_{3 k}(h)=-\gamma^{2} I_{n-r} .
\end{gathered}
$$

The following results can be obtained easily:

$$
\begin{gathered}
A^{T} P_{k}(h+1) A=E^{T} P_{k}(h) E-\gamma^{2} \widehat{N}^{-T} \widehat{N}^{-1}, \\
E^{T} P_{k}(k) E=E^{T} R E .
\end{gathered}
$$

And assume, by contradiction, that there exists $\bar{x} \in R^{n}$ such that

$$
\bar{x}^{T} E^{T} P_{k}(0) E \bar{x}>\varepsilon^{2} \bar{x}^{T} E^{T} R E \bar{x} .
$$

Again let $x(0)=\lambda \bar{x}$ such that $x^{T}(0) E^{T} R E x(0)=1$ and $x(k)$ is the state evolution starting from $x(0)$. By (18) it follows that

$$
x^{T}(k) E^{T} P_{k}(k) E x(k)-x^{T}(0) E^{T} P_{k}(0) E x(0)+\|z\|_{2}^{2}=0,
$$

from which it follows that

$$
x^{T}(k) E^{T} R E x(k)+\|z\|_{2}^{2}=x^{T}(0) E^{T} P_{k}(0) E x(0)>\varepsilon^{2},
$$

which contradicts (15). Therefore we have proven that, for any $k \in\{0,1,2, \ldots, N\}$, there exists

$$
P_{k}(\cdot): h \in\{0,1,2, \ldots, k\} \longmapsto P_{k}(h) \in R^{n \times n},
$$

such that (6a), (6b), and (6c) hold, and then the proof of Theorem 7 is complete.

Remark 8. In the case of $E=I$; that is, the DTLSS (1) reduces to a state-space one, Theorem 7 coincides with the well-known FTS results of discrete-time state-space systems [14], from this point of view, and Theorem 7 naturally extends existing results on discrete-time state-space systems to singular ones. 
Furthermore, as pointed out that Theorem 7 is without assuming the regularity of system (1), and it is also given in terms of the coefficient matrices of whole system, the matrix $P_{k}(\cdot)$ in $(6 \mathrm{a}),(6 \mathrm{~b})$, and $(6 \mathrm{c})$ is not positive definite matrix which results in difficulty in computation; thus, the application of Theorem 7 is not convenient. The result of the following is to remove such inequalities and establish other matrix inequality conditions.

Theorem 9. The DTLSS (1) is regular, causal, and FTS with respect to $(\varepsilon, R, N)$, if and only if, for each $k \in\{1,2, \ldots, N\}$, there exist a symmetric matrix $S$ and a positive definite matrixvalued function

$$
X_{k}(\cdot): h \in\{0,1,2, \ldots, k\} \longmapsto X_{k}(h) \in R^{n \times n},
$$

such that

$$
\begin{gathered}
A^{T}\left[X_{k}(h+1)-\Phi S \Phi^{T}\right] A-E^{T} X_{k}(h) E<0, \\
h \in\{0,1,2, \ldots, k-1\}, \\
E^{T} X_{k}(k) E \geq E^{T} R E, \\
E^{T} X_{k}(0) E \leq \varepsilon^{2} E^{T} R E,
\end{gathered}
$$

where $\Phi \in R^{n \times(n-r)}$ is any matrix with full column and satisfies $E^{T} \Phi=0$.

Proof. Consider the following.

Sufficiency. The inequalities in (6a), (6b), and (6c) are obviously satisfied by setting $P_{k}(h)=\left(X_{k}(h)-\Phi S \Phi^{T}\right)$ in $(25 \mathrm{a})$, (25b) and (25c) and we conclude that the DTLSS (1) is FTS with respect to $(\varepsilon, R, N)$ by Theorem 7 .

Necessity. Assume that the DTLSS (1) is FTS with respect to $(\varepsilon, R, N)$, then system (1) is regular, causal, and there are nonsingular matrices $\widehat{M}, \widehat{N} \in R^{n \times n}$ such that (14).

Let $x(k)=\widehat{N}\left(\frac{\bar{x}_{r}(k)}{\bar{x}_{2}(k)}\right)$; then system (1) is equivalent to

$$
\left(\begin{array}{cc}
I_{r} & 0 \\
0 & 0
\end{array}\right)\left(\begin{array}{ll}
\bar{x}_{r} & (k+1) \\
\bar{x}_{2} & (k+1)
\end{array}\right)=\left(\begin{array}{cc}
A_{r} & 0 \\
0 & I_{n-r}
\end{array}\right)\left(\begin{array}{ll}
\bar{x}_{r} & (k) \\
\bar{x}_{2} & (k)
\end{array}\right),
$$

where $A_{r}$ is $R^{r \times r}$.

Noting

$$
x^{T}(h) E^{T} R E x(h)=\bar{x}_{r}^{T}(h)\left(\begin{array}{ll}
I_{r} & 0
\end{array}\right)\left(\widehat{M}^{-T} R \widehat{M}^{-1}\right)\left(\begin{array}{c}
I_{r} \\
0
\end{array}\right) \bar{x}_{r}(h),
$$

that is, by Lemma 6 , system $\bar{x}_{r}(k+1)=A_{r} \bar{x}_{r}(k)$ is FTS with respect to $\left(\varepsilon,\left(\begin{array}{ll}I_{r} & 0\end{array}\right)\left(\widehat{M}^{-T} R \widehat{M}^{-1}\right)\left(\begin{array}{c}I_{r} \\ 0\end{array}\right), N\right)$ and the following result can be easily established. This fact implies that, for each $k \in\{1,2, \ldots, N\}$, there exists a symmetric matrix-valued function

$$
P_{r k}(\cdot): h \in\{0,1,2, \ldots, k\} \longmapsto P_{r k}(h) \in R^{n \times n},
$$

such that

$$
\begin{aligned}
& A_{r}^{T} P_{r k}(h+1) A_{r}-P_{r k}(h)<0, \quad h \in\{0,1,2, \ldots, k-1\}, \\
& P_{r k}(k) \geq\left(\begin{array}{ll}
I_{r} & 0
\end{array}\right)\left(\widehat{M}^{-T} R \widehat{M}^{-1}\right)\left(\begin{array}{c}
I_{r} \\
0
\end{array}\right), \\
& P_{r k}(0) \leq \varepsilon^{2}\left(\begin{array}{ll}
I_{r} & 0
\end{array}\right)\left(\widehat{M}^{-T} R \widehat{M}^{-1}\right)\left(\begin{array}{c}
I_{r} \\
0
\end{array}\right) .
\end{aligned}
$$

Rewrite the inequality above in the following form:

$$
\begin{aligned}
& \left(\begin{array}{cc}
A_{r} & 0 \\
0 & I_{n-r}
\end{array}\right)^{T}\left(\begin{array}{cc}
P_{r k}(h+1) & 0 \\
0 & I_{n-r}
\end{array}\right)\left(\begin{array}{cc}
A_{r} & 0 \\
0 & I_{n-r}
\end{array}\right) \\
& -\left(\begin{array}{cc}
I_{r} & 0 \\
0 & 0
\end{array}\right)^{T}\left(\begin{array}{cc}
P_{r k}(h) & 0 \\
0 & I_{n-r}
\end{array}\right)\left(\begin{array}{cc}
I_{r} & 0 \\
0 & 0
\end{array}\right) \\
& -\left(\begin{array}{cc}
0 & 0 \\
0 & 2 I_{n-r}
\end{array}\right)<0, \quad h \in\{0,1,2, \ldots, k-1\}, \\
& \left(\begin{array}{cc}
I_{r} & 0 \\
0 & 0
\end{array}\right)^{T}\left(\begin{array}{cc}
P_{r k}(k) & 0 \\
0 & I_{n-r}
\end{array}\right)\left(\begin{array}{cc}
I_{r} & 0 \\
0 & 0
\end{array}\right) \\
& \geq\left(\begin{array}{cc}
\left(\begin{array}{cc}
I_{r} & 0
\end{array}\right)\left(\begin{array}{cc}
\widehat{M}^{-T} R \widehat{M}^{-1}
\end{array}\right)\left(\begin{array}{c}
I_{r} \\
0
\end{array}\right) & 0 \\
0 & 0
\end{array}\right) \\
& =\left(\begin{array}{cc}
I_{r} & 0 \\
0 & 0
\end{array}\right)^{T}\left(\widehat{M}^{-T} R \widehat{M}^{-1}\right)\left(\begin{array}{cc}
I_{r} & 0 \\
0 & 0
\end{array}\right),
\end{aligned}
$$

$$
\begin{aligned}
& \left(\begin{array}{cc}
I_{r} & 0 \\
0 & 0
\end{array}\right)^{T}\left(\begin{array}{cc}
P_{r k}(0) & 0 \\
0 & I_{n-r}
\end{array}\right)\left(\begin{array}{cc}
I_{r} & 0 \\
0 & 0
\end{array}\right) \\
& \leq \varepsilon^{2}\left(\begin{array}{cc}
\left(\begin{array}{ll}
I_{r} & 0
\end{array}\right)\left(\begin{array}{cc}
\widehat{M}^{-T} R \widehat{M}^{-1} \\
0
\end{array}\right)\left(\begin{array}{c}
I_{r} \\
0
\end{array}\right) & 0 \\
0 & 0
\end{array}\right) \\
& =\left(\begin{array}{cc}
I_{r} & 0 \\
0 & 0
\end{array}\right)^{T}\left(\widehat{M}^{-T} R \widehat{M}^{-1}\right)\left(\begin{array}{cc}
I_{r} & 0 \\
0 & 0
\end{array}\right) .
\end{aligned}
$$

Define

$$
\widehat{M}=\left(\begin{array}{l}
M_{1} \\
M_{2}
\end{array}\right), \quad Q_{k}(h)=\left(\begin{array}{cc}
P_{r k}(h) & 0 \\
0 & I_{n-r}
\end{array}\right),
$$

where $M_{2} \in R^{(n-r) \times n}, M_{2} E=\left(\begin{array}{ll}0 & 0\end{array}\right)$, and $M_{2} A \widehat{N}=\left(\begin{array}{ll}0 & I_{n-r}\end{array}\right)$.

Substituting (14) into the above inequality (30a), (30b), and (30c), we can conclude

$$
\begin{aligned}
& \widehat{N}^{T} A^{T} \widehat{M}^{T} Q_{k}(h+1) \widehat{M} A \widehat{N}-\widehat{N}^{T} E^{T} \widehat{M}^{T} Q_{k}(h) \widehat{M} E \widehat{N} \\
& \quad-\widehat{N}^{T} A^{T} M_{2}^{T} W^{T} W^{-T}\left(2 I_{n-r}\right) W^{-1} W M_{2} A \widehat{N}<0, \\
& \widehat{N}^{T} E^{T} \widehat{M}^{T} Q_{k}(k) \widehat{M} E \widehat{N} \\
& \geq \widehat{N}^{T} E^{T} \widehat{M}^{T}\left(\widehat{M}^{-T} R \widehat{M}^{-1}\right) \widehat{M} E \widehat{N}=\widehat{N}^{T} E^{T} R E \widehat{N}, \\
& \widehat{N}^{T} E^{T} \widehat{M}^{T} Q_{k}(0) \widehat{M} E \widehat{N} \\
& \leq \varepsilon^{2} \widehat{N}^{T} E^{T} \widehat{M}^{T}\left(\widehat{M}^{-T} R \widehat{M}^{-1}\right) \widehat{M} E \widehat{N}=\varepsilon^{2} \widehat{N}^{T} E^{T} R E \widehat{N} .
\end{aligned}
$$


Define

$$
\begin{gathered}
X_{k}(h)=\widehat{M}^{T} Q_{k}(h) \widehat{M}, \quad \Phi^{T}=W M_{2}, \\
S=2 W^{-T} I_{n-r} W^{-1} .
\end{gathered}
$$

We can conclude

$$
\begin{aligned}
& \widehat{N}^{T} A^{T} X_{k}(h+1) A \widehat{N}-\widehat{N}^{T} E^{T} X_{k}(h) E \widehat{N} \\
&- \widehat{N}^{T} A^{T} \Phi S \Phi^{T} A \widehat{N}<0, \\
& \widehat{N}^{T} E^{T} X_{k}(k) E \widehat{N} \geq \widehat{N}^{T} E^{T} R E \widehat{N}, \\
& \widehat{N}^{T} E^{T} X_{k}(0) E \widehat{N} \leq \varepsilon^{2} \widehat{N}^{T} E^{T} R E \widehat{N},
\end{aligned}
$$

which is equivalent to (25a), (25b), and (25c). Thus, the proof is completed.

From Theorem 9, the following result can be easily established.

Corollary 10. If there exist a symmetric matrix $S$ and a positive definite matrix-valued function $X(k)$

$$
X(\cdot): k \in\{0,1,2, \ldots, N\} \longmapsto X(k) \in R^{n \times n},
$$

such that

$$
\begin{gathered}
A^{T}\left[X(k+1)-\Phi S \Phi^{T}\right] A-E^{T} X(k) E<0, \\
k \in\{0,1,2, \ldots, N-1\}, \\
E^{T} X(k) E>E^{T} R E, \\
E^{T} X(0) E<\varepsilon^{2} E^{T} R E,
\end{gathered}
$$

where $\Phi \in R^{n \times(n-r)}$ is any matrix with full column and satisfies $E^{T} \Phi=0$. Then the DTLSS (1) is regular, causal, and FTS with respect to $(\varepsilon, R, N)$.

Proof. It is straightforward to check that a matrix function $X(\cdot)$ satisfies conditions (36a), (36b), and (36c) also satisfying conditions (25a), (25b), and (25c).

Remark 11. Two necessary and sufficient conditions of FTS for discrete-time singular systems are given, which can be expressed in terms of matrix inequalities, the application of them is not convenient. The sufficient conditions for FTS in corollary are in the form of LMIs; they can be solved by Matlab software easily.

\section{Illustrative Example}

In this section, an example is presented to demonstrate the applicability of proposed approach.

Consider the discrete-time singular system (1) with parameters as follows:

$$
E=\left(\begin{array}{ll}
1 & 0 \\
0 & 0
\end{array}\right), \quad A=\left(\begin{array}{cc}
2.5 & 1 \\
1 & 1
\end{array}\right) .
$$

It is easy to show that the system is not LAS. Now, we chose $R=I$ and $N=4$ and decide to perform an optimization over $\varepsilon$ with the aid of Matlab LMI Toolbox. We solved the LMIs (36a), (36b), and (36c) by Corollary 10 and we found guarantees that the desired system properties with $\varepsilon=5.0625$.

\section{Conclusion}

This paper has dealt with the finite-time stability problem of discrete-time linear singular systems. Two necessary and sufficient conditions for FTS are obtained without resorting decomposing the system matrices, which can be expressed in terms of matrix inequalities. However, such conditions are numerically hard to solve. Therefore a sufficient condition, checking the feasibility of only one inequality that requires the computation of the solution of a certain Lyapunov matrix inequality and can be expressed in terms of LMIs, has been given. An example illustrates the effectiveness of the proposed technique. Further research will focus on the problem of controller design.

\section{Conflict of Interests}

The authors declare that there is no conflict of interests regarding the publication o this paper.

\section{References}

[1] S. Xu, C. Yang, Y. Niu, and J. Lam, "Robust Stabilization for uncertain discrete singular systems: a matrix inequalities approach," Automatica, vol. 37, no. 5, pp. 769-774, 2001.

[2] L. Zhang, J. Lam, and Q. Zhang, "Lyapunov and Riccati equations of discrete-time descriptor systems," IEEE Transactions on Automatic Control, vol. 44, no. 11, pp. 2134-2139, 1999.

[3] Q. L. Zhang, W. Q. Liu, and D. Hill, "A Lyapunov approach to analysis of discrete singular systems," Systems \& Control Letters, vol. 45, no. 3, pp. 237-247, 2002.

[4] Z. Wu, H. Su, and J. Chu, "Delay-dependent $\mathrm{H}_{\infty}$ filtering for singular Markovian jump time-delay systems," Signal Processing, vol. 90, no. 6, pp. 1815-1824, 2010.

[5] Z.-G. Wu, P. Shi, H. Su, and J. Chu, " $I_{2}-I_{\infty}$ filter design for discrete-time singular Markovian jump systems with timevarying delays," Information Sciences, vol. 181, no. 24, pp. 55345547, 2011.

[6] P. Dorato, "Short time stability in linear time-varying systems," in Proceedings of IRE International Convention (Record Part 4), pp. 83-87, 1961.

[7] L. Weiss and E. F. Infante, "Finite time stability under perturbing forces and on product spaces," IEEE Transactions on Automatic Control, vol. 12, no. 1, pp. 54-59, 1967.

[8] F. Amato, M. Ariola, and P. Dorato, "Finite-time control of linear systems subject to parametric uncertainties and disturbances," Automatica, vol. 37, no. 9, pp. 1459-1463, 2001.

[9] F. Amato, M. Ariola, and C. Cosentino, "Finite-time stabilization via dynamic output feedback," Automatica, vol. 42, no. 2, pp. 337-342, 2006.

[10] E. Moulay and W. Perruquetti, "Finite time stability and stabilization of a class of continuous systems," Journal of Mathematical Analysis and Applications, vol. 323, no. 2, pp. 1430-1443, 2006. 
[11] G. Garcia, S. Tarbouriech, and J. Bernussou, "Finite-time stabilization of linear time-varying continuous systems," IEEE Transactions on Automatic Control, vol. 54, no. 2, pp. 364-369, 2009.

[12] F. Amato and M. Ariola, "Finite-time control of discrete-time linear systems," IEEE Transactions on Automatic Control, vol. 50, no. 5, pp. 724-729, 2005.

[13] F. Amato, R. Ambrosino, M. Ariola, and F. Calabrese, "Finitetime stability analysis of linear discrete-time systems via polyhedral lyapunov functions," in Proceedings of the American Control Conference (ACC '08), pp. 1656-1660, June 2008.

[14] F. Amato, M. Ariola, and C. Cosentino, "Finite-time control of discrete-time linear systems: analysis and design conditions Finite-time output feedback control of discrete-time systems," Automatica, vol. 46, no. 5, pp. 919-924, 2010.

[15] L. Zhu, Y. Shen, and C. Li, "Finite-time control of discrete-time systems with time-varying exogenous disturbance," Communications in Nonlinear Science and Numerical Simulation, vol. 14, no. 2, pp. 361-370, 2009.

[16] R. Ambrosino, F. Calabrese, C. Cosentino, and G. De Tommasi, "Sufficient conditions for finite-time stability of impulsive dynamical systems," IEEE Transactions on Automatic Control, vol. 54, no. 4, pp. 861-865, 2009.

[17] S. Zhao, J. Sun, and L. Liu, "Finite-time stability of linear timevarying singular systems with impulsive effects," International Journal of Control, vol. 81, no. 11, pp. 1824-1829, 2008.

[18] X. Zhang, G. Feng, and Y. Sun, "Finite-time stabilization by state feedback control for a class of time-varying nonlinear systems," Automatica, vol. 48, no. 3, pp. 499-504, 2012.

[19] J. Yin, S. Khoo, Z. Man, and X. Yu, "Finite-time stability and instability of stochastic nonlinear systems," Automatica, vol. 47, no. 12, pp. 2671-2677, 2011.

[20] C. A. Desoer and M. Vidyasagar, Feedback Systems: InputOutput Properties, Academic Press, New York, NY, USA, 1975.

[21] L. Dai, Singular Control System, vol. 118 of Lecture Notes in Control and Information Sciences, Springer, Berlin, Germany, 1989. 


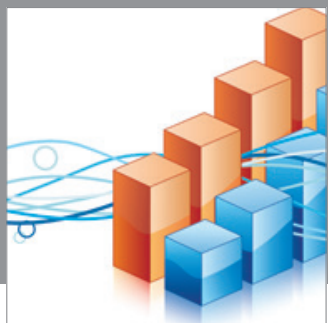

Advances in

Operations Research

mansans

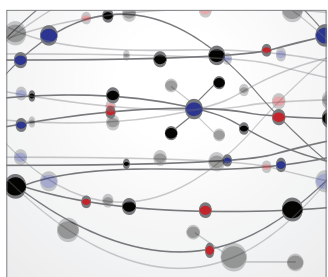

The Scientific World Journal
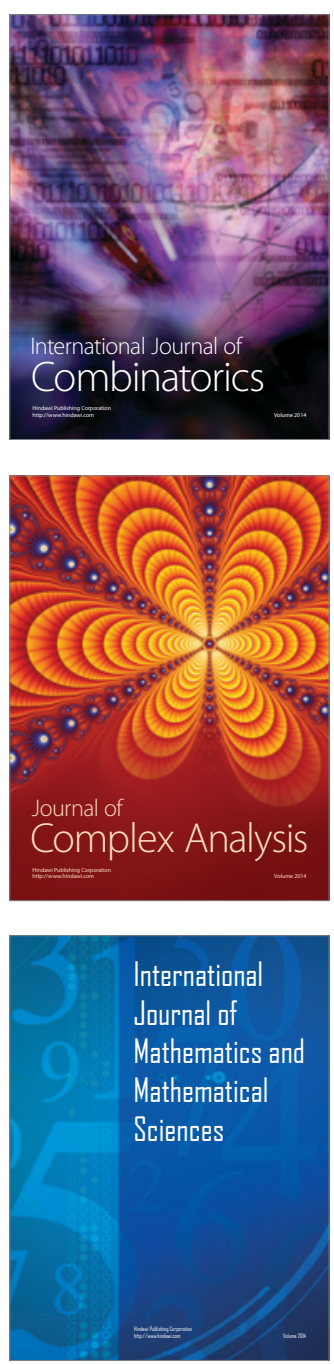
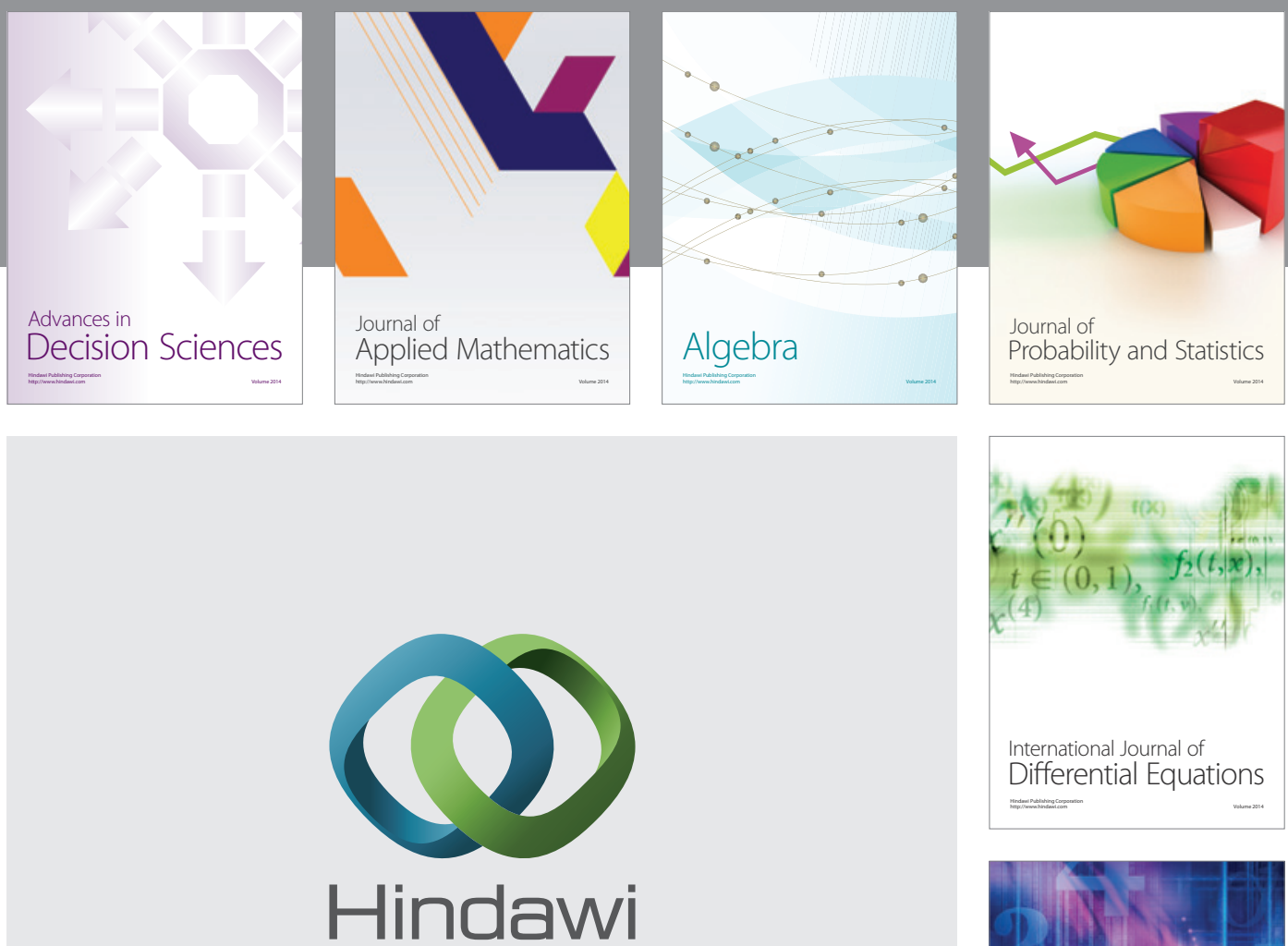

Submit your manuscripts at http://www.hindawi.com
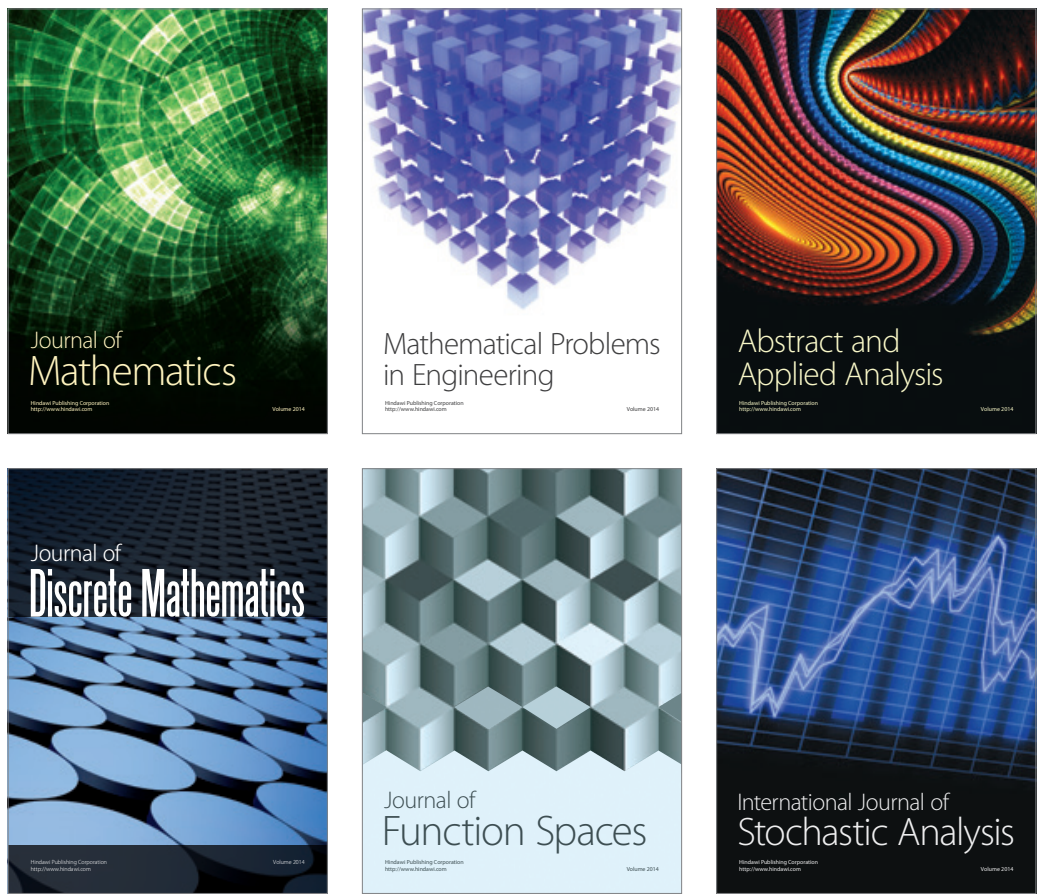

Journal of

Function Spaces

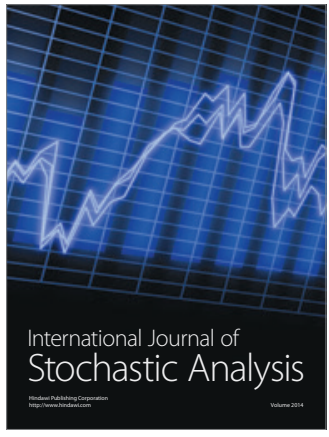

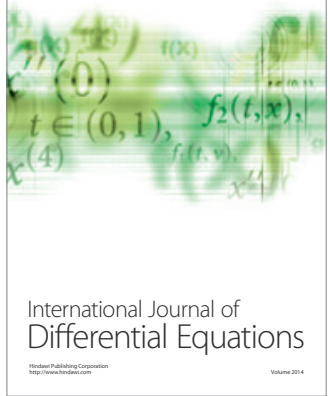
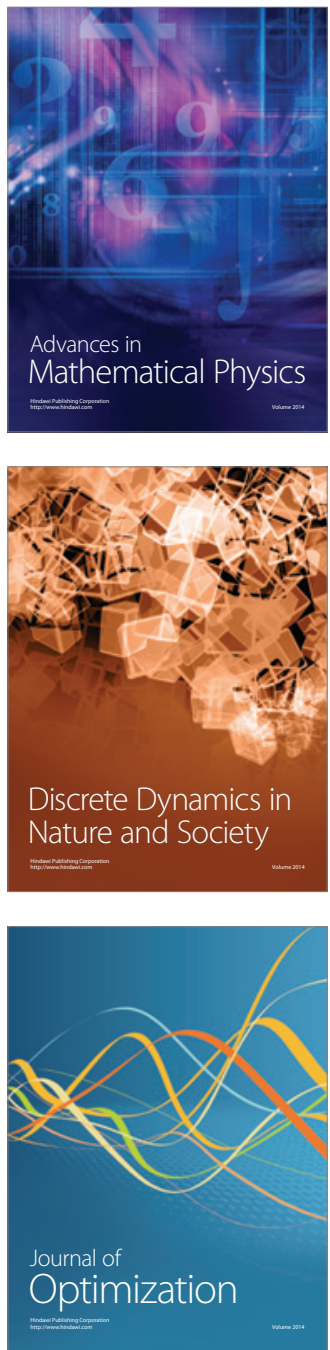